\title{
Accidentes o lesiones no intencionales: una perspectiva social
}

\section{Accidents or unintentional injuries: A social perspective}

\author{
Arturo Franco Ordaz y Rosa Elizabeth Sevilla Godinez
}

Universidad de Guadalajara $^{1}$

Autor para correspondencia: Arturo Franco Ordaz, psicofranco93@gmail.com.

\begin{abstract}
RESUMEN
Las lesiones no intenciones son conocidas popularmente como "accidentes", pero el designarlas como tales implica conceptualmente que son situaciones que están fuera del control humano. Popularizar conceptos que reasignen la capacidad de prevenir las lesiones ante los indicios de riesgo permite crear medidas que pueden evitar las consecuencias lesivas de ignorar el riesgo, y ver a así los llamados "accidentes" como fenómenos controlables, prevenibles y hasta eliminables.

Palabras clave: Riesgo; Lesión no intencional; Accidente; Peligro; Consecuencia; Detrimento; Prevención.
\end{abstract}

\begin{abstract}
Unintentional injuries are popularly known as "accidents", but designating them as such conceptually implies that such injuries are situations beyond human control. To promote and popularize the concept of unintentional injuries as involving the ability to prevent them on the basis of risk clues would help create interventions to avoid the harmful consequences of ignoring such risk. Thus it is necessary to conceive the so-called "accidents" as controllable, preventable, and even susceptible of elimination.
\end{abstract}

Keywords: Risk; Unintentional injury; Accident; Danger; Consequence; Detriment; Prevention.

Recibido: $13 / 01 / 2020$

Aceptado: $12 / 05 / 2020$

\section{INTRODUCCIÓN}

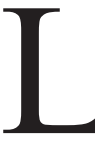

a forma en que se entiende "accidente" como concepto, o la interpretación que se le atribuye, influye en el comportamiento y en la actitud que se asume para vivir las situaciones ligadas al mismo, es decir, como debidas al azar. En cambio, el término de "lesiones no intencionales", no implican una incapacidad para controlarlas, sino el daño causado sin insidia y la posibilidad de que su prevención sea factible; sin

\footnotetext{
${ }^{1}$ Centro Universitario de Ciencias de la Salud, Sierra Mojada 950, Col. Independencia Oriente, 44340 Guadalajara, Jal., México, tel.(333)397-91-63, correos electrónicos: psicofranco93@gmail.com y elizros@hotmail.com.
} 
embargo, la palabra a la que se recurre de forma coloquial para denotarlas es la ya mencionada "accidente", que es una consecuencia del riesgo. No obstante, tal término causa problemas en la salud pública ya que denota la imposibilidad de actuar frente al riesgo o el peligro para prevenirlo o evitarlo (Ruiz, 2011). En el Diccionario de la Real Academia de la Lengua Española (2016) la palabra peligro se define como "riesgo o contingencia inminente de que suceda algún mal"; a su vez, riesgo es definido como "contingencia o proximidad de un daño". Según sus raíces epistemológicas (Corominas, 2003), "peligro" deriva del latín periculum, que hace referencia a un "ensayo" o "prueba", situando entonces la posible lesión en el futuro, y de la misma raíz peritus, que significa "experimentado". Por su parte, se cree que "riesgo" deriva de risco o rischio, del castellano "risco" o "peñasco escarpado", por el peligro de transitar por él.

Al realizar el análisis de las definiciones, los conceptos de "peligro" y "riesgo" se retroalimentan, pues ambos aluden a la posibilidad de sufrir un daño, lo que origina que las acciones o las consideraciones sobre la capacidad para impedir lesiones en un cierto evento se vean infravaloradas, ya que existe la posibilidad de evitar estas. El problema surge cuando se compara lo que se desea lograr y el posible perjuicio que se puede generar en el proceso. Se subestima la probabilidad e intensidad del daño latente porque se ha aprendido que las experiencias riesgosas pueden alcanzarse sin sufrir daños, o que estos pueden ser mínimos; es decir, constituyen un riesgo aceptable.

Es esta misma exposición repetitiva o prolongada lo que aumenta la probabilidad del daño; haber salido bien librados del peligro o con un daño mínimo en eventos anteriores lleva a creer que se puede volver al peligro eludiendo las consecuencias negativas y obteniendo lo que se desea.

El riesgo es visto como una probabilidad. Se asume la consecuencia de un posible daño, y a la par la capacidad de lograr el éxito eludiéndolo. Al parecer, la posibilidad de salir bien librados es lo que vuelve a la palabra "riesgo" un término sesgado que aumenta la probabilidad de que se actúe de forma inadecuada para evitar aquel. Es decir, el riesgo es en sí la capacidad del daño, y está presente en dos elementos que aparecen como posibles causas de ese riesgo: obtener lo deseado sin el daño, o afrontar el daño junto con lo deseado. Se puede percibir el riesgo como un daño, pero se aprecia también la posibilidad de tener éxito; por tanto, se hace la comparación entre el costo y el beneficio de tomar el riesgo, entre la comodidad de elegir el riesgo y la ejecución de tareas más elaboradas que lo anulen. Cuando la mayor parte de las veces se logra lo que se busca, es cuando se tiende a seguir asumiendo el riesgo que ello implica, creyendo que el azar es favorable. Es de esta forma que el riesgo proporciona una falsa libertad de asumir la responsabilidad al considerarse como algo incontrolable, a la vez que le resta importancia al daño por ser solo una posibilidad más en una situación de riesgo. Y esto es así porque la mayoría de las veces al asumir el riesgo se tiene éxito evadiendo el daño, lo que hace creer que la posibilidad de enfrentar al peligro y resultar dañado es mínima o nula, lo que genera una especie de experticia en la actividad de riesgo y una actitud poco o nada precavida. Del control y la atención al peligro se pasa a ignorarlo, a no esperarlo, llegando entonces a anular, dentro de las creencias, las posibilidades de que ocurra, a ocultarlo y a la vez potenciarlo, y a sorprendernos de que no lo hubiéramos considerado cuando ya no tomamos las precauciones que permitirían evadir o disminuir el daño que trajo consigo.

\section{El fatal riesgo}

Evadir la capacidad del daño es pasar de su desestimación a su anulación, convirtiéndose de este modo en un riesgo latente, invisible y parte de la vida diaria. Se habla del fatalismo como la aceptación de un presente y un futuro ineludiblemente trágico, que depende del universo o de un ser divino que se halla fuera de nuestros límites; es decir, es una actitud de desigualdad frente al daño: ya no importa el peligro pues es algo en lo que se está sumergido y del cual ya no importa escapar (Blanco y Díaz, 2007). A esto, Martín-Baró (1989) afirma que tal estado de desinterés, de fatalismo, parte de la construcción social de pobreza, 
explotación y desigualdad social, contexto que se vive principalmente en Latinoamérica. Dicha actitud está conformada por creencias, sentimientos y comportamientos. Martín-Baró señala tres principales creencias «1. "La vida está predefinida"; 2. "La propia acción no puede cambiar el destino fatal", y 3. "Un Dios lejano y todopoderoso decide el destino de cada persona"» (p. 157), mismas que llevan a la inactividad, a dejar de pensar y planear un futuro, a vivir un presente constante sin consecuencias controlables, a la falta de conciencia de que la acción tiene efectos venideros. El fatalismo crea un círculo vicioso de conformismo al negar de forma ilusoria la existencia del riesgo: no se actúa para eliminarlo; por el contrario, perpetuando el daño posible, se aumenta su probabilidad. Asumir el conformismo como un problema cultural o de orden público parte de una perspectiva sociocultural (Frías, 2006); es decir no depende el riesgo totalmente de las características físicas del medio o de las estructuras cognitivas de los individuos de forma particular, sino que, bajo esta perspectiva, la valoración del riesgo depende de la construcción social vinculada a estructuras dadas de los valores sociales y la confianza que se tiene en las instituciones al representar una autoridad de conocimiento, así como de la manera en que se transforma la información en los medios. Debatir el concepto de riesgo y verlo como una problemática social (Sastre, Zonib, Esparza y Del Cura, 2016) es una forma de vislumbrar la dificultad cuando se emplean los términos "riesgo" y "peligro", tan coloquialmente usados para referirse a las lesiones no intencionales. Es también una invitación a buscar un nuevo concepto capaz de romper con la posibilidad y que conlleve una prevención adecuada, además de motivar a las personas a tomar medidas preventivas por la seguridad que brindan, tanto de salud como de éxito en lo que se pretende lograr, separando así el concepto de riesgo de sus consecuencias negativas, esto es, las lesiones no intencionales.

\section{Consumir el riesgo}

Pareciera que en la cultura de consumismo, de competencia, en la que se debe hacer más que el otro, o incluso hacer más cada día en lo individual, es lo que da un valor, el que se obtiene en ocasiones a costa del daño al sujeto mismo o hacia los demás (Pacheco, 2012). En el caso del riesgo, es una fuente de reafirmación de las habilidades y de la capacidad de superar los problemas de la vida cotidiana, entre ellos el peligro, el riesgo vivido (Calvario, 2014). En otras palabras, el hecho de asumir o tomar el riesgo nos hace parecer como personas valientes y nos da una sensación de control, de poder. Una cultura donde se tiene que asumir y superar los obstáculos, donde los roles de género han creado la idea de que para ser "hombres" hay que ser arriesgados, y aquel que no lo haga se ve denostado como femenino; incluso las mujeres se adaptan para sumarse a esta idea al asumir roles propios de los hombres: "arriesgadas, valientes, temerarias y agresivas" (Lagarde y De los Ríos, 2008).

La idea es siempre ser el que logra más y demostrar que se es mejor que el otro, incluso mejor que uno mismo progresivamente. Acaparar recursos y capacidades parece ser el objetivo de tomar riesgos, los que se vuelven una prueba para demostrar la competencia de la persona. Con base en la actitud competitiva, prevenir es demostrar la incapacidad de superar los obstáculos; además, aparece como la premisa latente del capitalismo: la idea de la economía aplicada a la prevención; ahorrar recursos para afrontar riesgos, subrayar el costo-beneficio, el gasto que implica evitar un daño que realmente no parece seguro, constituye una inversión peligrosa, quizá con menos beneficios que gastos en términos de dinero, tiempo y esfuerzos físicos y cognitivos (Acevedo, 2013). No parece ser entonces la prevención una medida conveniente desde la perspectiva del consumidor del riesgo, para quien esta "adquisición" lo reafirma socialmente como "poseedor", igual o superior que los demás en el continuo competir. Sin embargo, desde una perspectiva empresarial con una formación adecuada en la prevención, se opta por no tener pérdidas y no tanto por ganar más (Bestratén y Salas, 2013). Aunque esta postura parece contraria, proviene del mismo sistema enfocado al capital, pues una posición que se puede aplicar a los riesgos de lesiones no es buscar más salud, sino protegerla en el momento adecuado. 


\section{La prevención del daño}

Las campañas de prevención promueven la idea de realizar un esfuerzo más allá del acostumbrado por quienes no toman las precauciones debidas, sin considerar que estas personas no ven en esta actividad extra una gratificación convencional (la reafirmación de su persona o bien algún valor económico). Pareciera que en esas campañas se promociona la capacidad de deslindarse de la responsabilidad y de ignorar todo control sobre el riesgo $^{2}$, sin tomar en cuenta las medidas adecuadas en casos de peligro; por ejemplo, los padres o tutores en el cuidado de menores de edad. Este deslinde de la responsabilidad y la falta de control sobre el riesgo lleva en ocasiones a la sociedad a acusar al sistema de no proporcionar los recursos suficientes, o bien a culpar a alguna divinidad de la fatalidad del destino. Las campañas de concientización y prevención del riesgo en los anuncios televisivos o espectaculares, señalizaciones, spots de radio, talleres y charlas, son herramientas para concientizar a las personas y hacer que el daño no ocurra. La Figura 1 muestra la relación de causas de inconsciencia y consciencia del riesgo, siendo el resultado "las campañas de prevención", con las consideraciones que se tratan de reflejar a través de este texto.

Figura 1. El riesgo y sus causas.

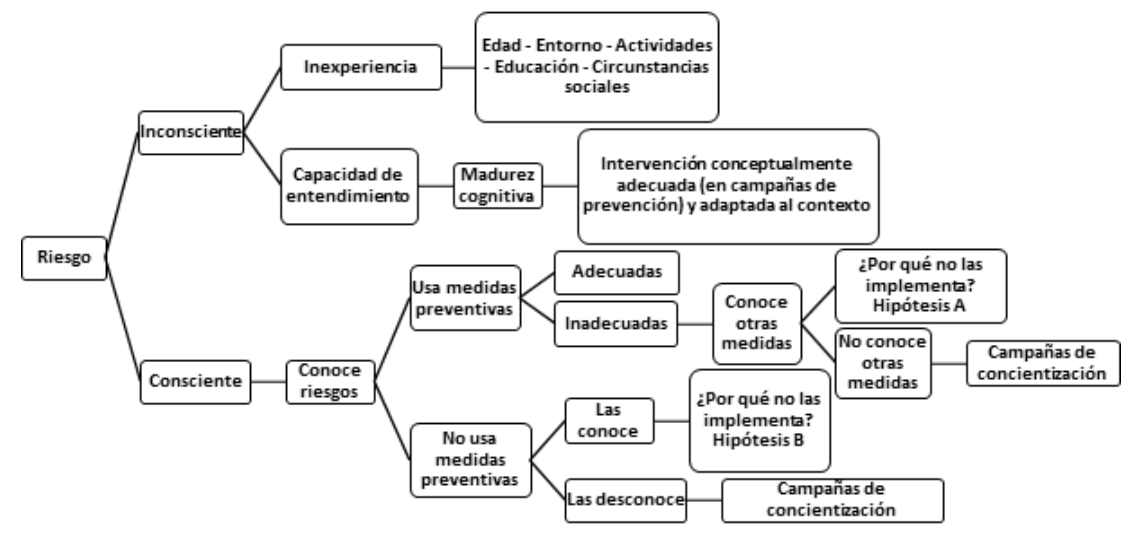

La Figura 2 muestra algunas hipótesis explicativas sobre el fatalismo como apaciguador de la acción $\mathrm{y}$ de asumir el riesgo como una prueba derivada de la idea capitalista; la minimización del riesgo refleja las consecuencias de asumir el riesgo y del fatalismo.

Figura 2. Hipótesis de la toma de riesgo.

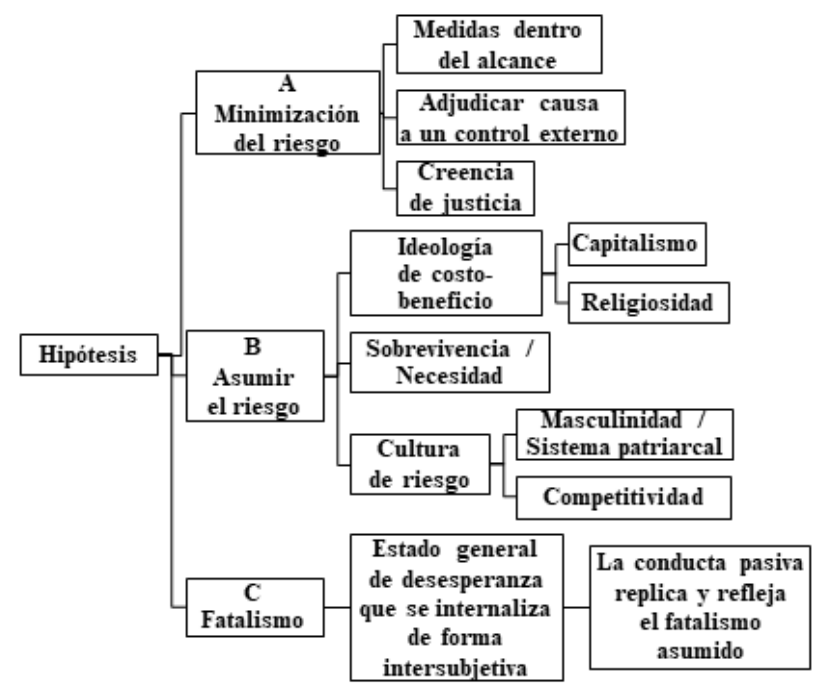

\footnotetext{
${ }^{2}$ De hecho, cuando se usa el término "accidente" dentro de los slogans, si bien se usa en ocasiones el de "lesión no intencional", este no forma parte de publicidad, sino que está en el contenido de los programas elaborados para las campañas.
} 
Como parte de la minimización del riesgo existen creencias que ven las lesiones como justas debido al comportamiento, ya como consecuencia de actuar de forma precipitada, ya como un castigo de alguna fuerza superior, generalmente de alguna divinidad, por lo que parcialmente pudiera considerarse como una actitud fatalista. Las campañas llegan a las personas de una forma que puede parecer inadecuada. Los términos que usan ofrecen comodidad al eliminar o reducir responsabilidad de las creencias socialmente construidas. Se asumen esos términos y se reflejan en el comportamiento de riesgo. Es necesario utilizar los medios de comunicación, las campañas y métodos de concientización sobre el riesgo y la prevención del daño empleando nuevos términos que impliquen realmente que las consecuencias de asumir riesgos - ya sean como reto o como algo que no se puede evitar- existen, están presentes y pueden ser irreversibles e incluso mortales. Quizá sea momento de dejar de hablar de "accidentes" y sustituir el término por lesiones no intencionales, y emprender campañas no de prevención como tal en un primer momento, sino de concientizar a las personas que no son "accidentes", sino lesiones no intencionales, para programar en el pensamiento colectivo la posibilidad de realizar acciones para disminuir su incidencia.

Si bien modificar los términos usados en esas campañas para prevenir y concientizar sobre los riesgos no cambia las estructuras y las actitudes sociales, sí permite no seguir promoviendo la concepción de la escasa capacidad de control que se tiene para evitarlos, y modificar la acción directa reflexionando el lugar de las personas como agentes capaces de prevenirlos, lo que contribuiría a eliminar la actitud fatalista y fomentar la visión del riesgo no como una prueba a superar, sino como algo a evitar.

\section{Lesiones no intencionales}

La forma adecuada de identificar el riesgo y sus consecuencias en conjunto, así como ver las lesiones, llamadas comúnmente "accidentes" como resultado directo de las medidas de prevención inadecuadas, es mediante el término lesión no intencional, el cual permite replantear la forma de apreciar los accidentes (Ruiz, 2011). Asimismo, desaparece conceptualmente la capacidad del azar, ligada a términos tales como "accidente", "riesgo" o "peligro". Si se replantea la forma de nombrar la situación o hecho, para su prevención se puede hablar de evitar la lesión no intencional, y así trabajar directamente sobre el riesgo, siendo el objetivo actuar de forma preventiva y no sobre las consecuencias del descuido (Onís, Varona, Gil, Felici y Embid, 2015) lo que contribuye a transformar las probabilidades del daño en una forma de control sobre el riesgo, a fin de preverlo y generar estrategias adecuadas, cambiar el enfoque de la perspectiva del sentido de prevención de forma proactiva y actuar para mantener la seguridad, no para evitar el riesgo.

El término "detrimento" es señalado como una alternativa más para aludir a los daños generados por el riesgo y la falta de prevención adecuada. Si bien es una alternativa que hace referencia al campo de la economía debido a la adopción del término por dicha disciplina, la palabra en sí no es exclusiva de esa área. En la salud pública es usada en un contexto socioeconómico referido a los determinantes sociales y de la salud (Palomino, Grande y Linares, 2014). Sumado a esto, dicho término está excluido del uso común, y es por ello que se prefiere abundar y difundir el de "lesión no intencional", que aparece como una oportunidad para recuperar y reforzar la conciencia de control sobre el riesgo mediante medidas adecuadas de prevención y así deslindarlo del azar.

Si bien el contexto social ha llevado a ver el futuro - englobando las acciones y medidas preventivas frente al riesgo- como algo incontrolable, lo cual no tiene caso intentar resolver (Casado y Moreno, 2015), el cambio de lenguaje en el ámbito académico, que intenta popularizar el multicitado término, permitirá replantear la capacidad de actuar, la cual es la principal limitante que plantea el fatalismo en el que viven aquellas personas vulnerables económica, educativa y socioculturalmente.

\section{Conclusiones}

Este estado paradójico entre la idea del cuidado, la prevención y la salud se contrapone a la conducta 
demostrada por las personas, que parecen competir y arriesgarse sin una razón aparente, lo que ha llevado a crear las figuras ya mostradas, las que pretenden presentar de una forma lógica las creencias y modos de vivir que conducen a las personas a asumir riesgos. Lo anterior obliga a formular hipótesis en el orden social, es decir, que denotan que no son situaciones de casos particulares, sino conductas y circunstancias que se repiten en cualquier población. La minimización del riesgo como un resultado del comportamiento y su repetición constante implica la falta del cuidado adecuado.

Por otra parte, asumir el riesgo es la imagen del sistema socioeconómico que modifica a los sujetos al replicar ideas individualistas y competitivas. El fatalismo bien puede englobar las otras hipótesis, pero es presentado a la par como una propuesta teórica en sí misma que alude a una consecuencia de las desigualdades sociales existentes, que llevan a un estado tal de desinterés que se pierde el control y voluntad para realizar acciones preventivas.

Para la prevención de lesiones no intencionales es necesario reestructurar las campañas de concientización desde sus propios conceptos, si bien los estudios en torno al riesgo ya han modificado los términos como el de "accidente", que limitan la capacidad de actuar y el deslinde de las responsabilidades que implica el cuidado, falta llegar a la población en riesgo mediante conceptos adecuados y al nivel de cada sector.

Es cierto que se vive socialmente en un estado paradójico de competencia y, a la vez, de pasividad, esta última a causa de un fatalismo generado por el estado socioeconómico y cultural que se vive. La práctica de los términos adecuados en el ámbito académico y profesional, así como los propios de la comunidad, se vuelve necesaria para dejar de replicar el conformismo sobre la forma de vivir.

Citación: Franco O., A. y Sevilla G., R. E. (2021). Accidentes o lesiones no intencionales: una perspectiva social. Psicología y Salud, 31(2), 249-254. https://doi.org/10.25009/pys.v31i2.2693.

\section{REFERENCIAS}

Acevedo R., R.A. (2013). El proceso de toma de decisiones: un modelo de economía. Munich Personal RePEc Archive, 31 de mayo, 1-21. Recuperado de https://mpra.ub.uni-muenchen.de/50890/1/MPRA_paper_50890.pdf.

Bestratén B., M. y Salas O., C. (2013). Análisis coste-beneficio en la acción preventiva (I): bases conceptuales. Notas Técnicas de Prevención, 983, 1-10.

Blanco, A. y Díaz, D. (2007). El rostro bifronte del fatalismo: fatalismo colectivista y fatalismo individualista. Psicothema, 19(4), 552-558.

Calvario P., J.E. (2014). Género y masculinidad. Juegos de poder y configuración del peligro en el poblado Miguel Alemán, Sonora. México, D.F.: El Colegio de México.

Casado Q., N. y Moreno J., P. (2015). El fatalismo como consecuencia del internamiento en prisión y su relación con otras variables psicosociales. Apuntes de Psicología, 33(2), 49-56.

Corominas, J. (1987). Breve diccionario etimológico de la lengua castellana. Madrid: Gredos.

Frías O., A. (2006). La cultura y las conductas de riesgo en adolescentes. Granada (España): Universidad de Granada.

Lagarde R., M. (2008). El feminismo en mi vida: Hitos, claves y topías. México: Gobierno del Distrito Federal e Instituto de las Mujeres del Distrito Federal.

Martín-Baró, I. (1989). Sistema, grupo y poder psicología social desde Centroamérica II. San Salvador: UCA Editores.

Onís G., E., Varona P., I., Gil P., M., Felici, C. y Embid P., P. (2015). Lesiones no intencionadas en el centro escolar: ¿de qué estamos hablando? Revista Pediatría de Atención Primaria, 17(68), 333-339.

Pacheco F., R.A. (2012). El capitalismo neoliberal y su sujeto. Teoría y Crtítica de la Psicología, 2, $113-125$.

Palomino M., P.A., Grande G., M.L. y Linares A., M. (2014). La salud y sus determinantes sociales. Desigualdades y exclusión en la sociedad del siglo XXI. Revista Internacional de Sociología, 72(Extra 1), 71-91.

Real Academia de la Lengua Española. (30 de Noviembre de 2016). Diccionario de la Real Academia Española. Madrid: RAE.

Ruiz P., M. (2011). ¿Se debe usar el término accidente en el ámbito de la investigación científica? Panacea, 12 (33), 84-88.

Sastre P., M., Zonib A., C., Esparza O., M.J. y Del Cura G., M.I. (2016). Prevalencia y factores asociados a lesiones no intencionales. Revista Pediatría de Atención Primaria, 18(71), 1-6. 